\title{
A Review of Data-Driven Decision-Making Methods for Industry 4.0 Maintenance Applications
}

\author{
Alexandros Bousdekis (D), Katerina Lepenioti, Dimitris Apostolou* (D) and Gregoris Mentzas \\ Information Management Unit (IMU), Institute of Communication and Computer Systems (ICCS), \\ National Technical University of Athens (NTUA), 157-80 Athens, Greece; albous@mail.ntua.gr (A.B.); \\ klepenioti@mail.ntua.gr (K.L.); gmentzas@mail.ntua.gr (G.M.) \\ * Correspondence: dapost@unipi.gr
}

Citation: Bousdekis, A.; Lepenioti, K.; Apostolou, D.; Mentzas, G. A Review of Data-Driven Decision-Making Methods for Industry 4.0 Maintenance Applications. Electronics 2021, 10, 828 https://doi.org/10.3390/electronics 10070828

Academic Editor: Carlos A. Iglesias

Received: 9 March 2021

Accepted: 25 March 2021

Published: 31 March 2021

Publisher's Note: MDPI stays neutral with regard to jurisdictional claims in published maps and institutional affiliations.

Copyright: (c) 2021 by the authors. Licensee MDPI, Basel, Switzerland. This article is an open access article distributed under the terms and conditions of the Creative Commons Attribution (CC BY) license (https:// creativecommons.org/licenses/by/ $4.0 /)$.
Abstract: Decision-making for manufacturing and maintenance operations is benefiting from the advanced sensor infrastructure of Industry 4.0, enabling the use of algorithms that analyze data, predict emerging situations, and recommend mitigating actions. The current paper reviews the literature on data-driven decision-making in maintenance and outlines directions for future research towards data-driven decision-making for Industry 4.0 maintenance applications. The main research directions include the coupling of decision-making with augmented reality for seamless interfacing that combines the real and virtual worlds of manufacturing operators; methods and techniques for addressing uncertainty of data, in lieu of emerging Internet of Things (IoT) devices; integration of maintenance decision-making with other operations such as scheduling and planning; utilization of the cloud continuum for optimal deployment of decision-making services; capability of decision-making methods to cope with big data; incorporation of advanced security mechanisms; and coupling decision-making with simulation software, autonomous robots, and other additive manufacturing initiatives.

Keywords: Internet of Things; intelligent decision-making; data analytics; big data; predictive maintenance

\section{Introduction}

The current trend of automation and data exchange in manufacturing is enabled by emerging technological advancements including the Internet of Things (IoT), cloud computing, and cyber-physical systems. This trend is often cited as "Industry 4.0", "smart manufacturing", and "digital factory" [1]. The large volume of data generated by manufacturing automation and sophisticated machines and sensors have been described in various reviews of industrial communication and data management systems, e.g., [2,3]. Predictive maintenance in particular is gaining a crucial role in cost reduction and business performance improvement [4]. Predictive maintenance utilizes heterogeneous data sources for detecting abnormal behaviors of equipment (diagnosis), predicting future failure modes (prognosis), and supporting decisions ahead of time (proactive decision-making) [5].

The need to support data-driven decision-making in Industry 4.0 has leveraged the development of new methods and algorithms aiming to support engineers in making optimal decisions about maintenance and operational actions [6,7]. To the best of our knowledge, this is the first literature review about data-driven decision-making algorithms for manufacturing maintenance operations. This paper starts by surveying prominent decision-making approaches for manufacturing maintenance operations, a well-studied application area, and analyses decision-making algorithms that are triggered by real-time, data-driven analytics. The analysis of the state of the art leads to a synthesis of research challenges and the definition of a research agenda for data-driven decision-making for Industry 4.0 maintenance applications. 


\section{Scope of the Literature Review}

In this section, we describe the scope of our literature review. First, we briefly discuss the role of decision-making in maintenance according to the Potential Failure and Functional Failure (P-F) interval and the recent technological advancements (Section 2.1). We also present other literature reviews related to decision-making algorithms in maintenance along with their contributions and limitations and we identify the need for the current literature review (Section 2.2). Finally, we present our methodology for the literature review (Section 2.3).

\subsection{The Role of Decision-Making in Manufacturing Maintenance}

Methods used for predictive maintenance can be classified into three categories [8]: (i) model-based, relying on the physical models of the equipment operation and the manufacturing process; (ii) knowledge-based, relying on expert knowledge and being addressed by knowledge management systems; and (iii) data-driven, relying on data analytics and machine learning algorithms. In this work, we focus on the data-driven methods for maintenance decision-making.

Condition monitoring, i.e., the process of monitoring the condition in order to identify a significant change that is indicative of a developing fault [9], is a major component of predictive maintenance [10]. During the last years, due to the emergence of Industry 4.0, condition monitoring techniques have evolved from visual inspections and manual analysis of datasets to high-frequency sensors generating real-time big data on several parameters such as vibration, temperature, and thermography. On the basis of these data, one can apply advanced data analytics techniques in order to handle the uncertainty due to the stochastic degradation process as well as the uncertainty in prognostic output and to support decisionmaking under time constraints. Decision-making in predictive maintenance indicates the phase that is triggered by data-driven, (near) real-time predictions (e.g., about future failure modes) in order to generate proactive recommendations about maintenance actions and plans that eliminate or mitigate the impact of the predicted failure.

An important and well-established principle of maintenance is the P-F curve, which is shown in Figure 1. The P-F curve indicates how a part of equipment starts being degraded to the point at which the forthcoming failure can be predicted (the potential failure point " $\mathrm{P}$ "). Thereafter, if it is not predicted and no suitable action is taken, it continues to deteriorate-usually at an accelerating rate-until it reaches the point of functional failure (Point "F") - this is known as the P-F interval [11]. The P-F interval allows for actions to be taken so as to avoid the forthcoming failure or provide the necessary remedies [8].

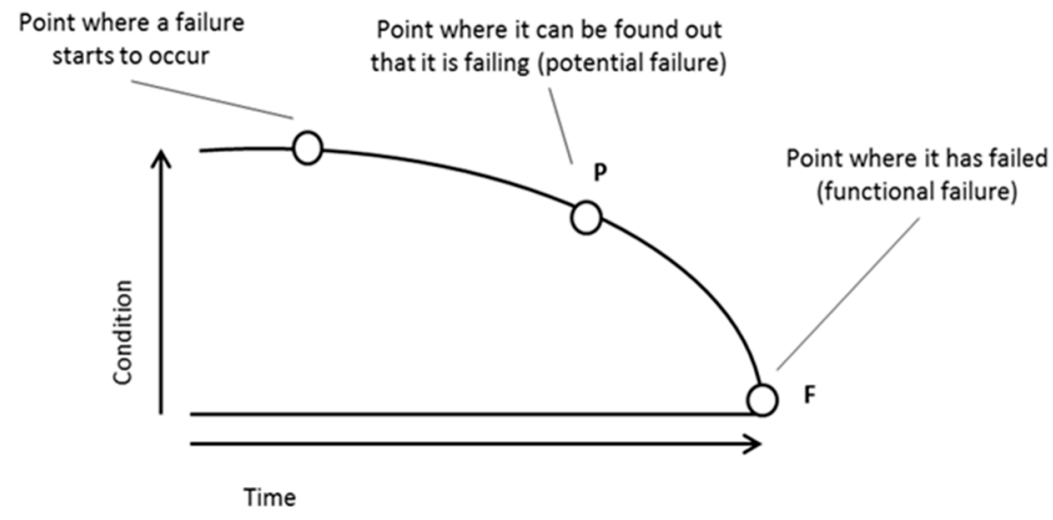

Figure 1. Potential Failure and Functional Failure (P-F) curve.

\subsection{Related Literature Reviews}

Table 1 shows related literature reviews published between 2011 and 2018 [6,12-15]. Each review has a specific contribution and focal point as outlined in the same table. Still, existing reviews have the following limitations: (i) they do not distinguish between 
static and dynamic models, e.g., offline and real-time models; (ii) decision-making is not necessarily triggered by data-driven analytics such as predictions; (iii) they focus on specific categories of decision methods, such as optimization, and/or maintenance aspects, such as maintenance policy; (iv) they are not matched to the Industry 4.0 pillars. Contrarily, our twork focuses on the review and analysis of data-driven decision-making methods that rely largely on data analytics and can enable emerging Industry 4.0 applications such as predictive maintenance.

\subsection{Methodology of the Literature Review}

The review protocol followed is based on the one presented by Tranfield et al. [16] and is shown in Table 2. We searched for research published after 2013, the year when the Working Group Industry 4.0 published their first report. Note that out review does not cover papers describing data-driven maintenance decision-making outside of the manufacturing or production environment, such as for end products or components, e.g., $[17,18]$, or urban facilities equipment, e.g., [19].

Table 1. Related reviews.

\begin{tabular}{|c|c|c|c|}
\hline Reference & Title & Contributions & Limitations \\
\hline [12] & $\begin{array}{c}\text { A literature review and future } \\
\text { perspectives on } \\
\text { maintenance optimization }\end{array}$ & $\begin{array}{l}\text { It reviews the literature on } \\
\text { methods and techniques for } \\
\text { maintenance optimization } \\
\text { models and associated } \\
\text { case studies. } \\
\text { It reveals emerging trends } \\
\text { (e.g., towards the use of } \\
\text { simulation for } \\
\text { maintenance optimization). }\end{array}$ & $\begin{array}{l}\text { It includes only } \\
\text { optimization models. } \\
\text { It deals with corrective, } \\
\text { preventive, and } \\
\text { predictive maintenance. } \\
\text { It does not distinguish } \\
\text { between static and } \\
\text { dynamic models. } \\
\text { Optimization is not necessarily } \\
\text { executed on the basis } \\
\text { of predictions. }\end{array}$ \\
\hline
\end{tabular}

- It presents a review of existing maintenance optimization models.

- It outlines the effectiveness of preventive and risk-based maintenance with respect to corrective maintenance.

Maintenance optimization models: a review and analysis optimization systems: a review
- It concludes that most of the literature addresses optimization solutions in static environments. Thus, it outlines the importance of dynamic models.
- It includes only optimization models.

- It deals with corrective, preventive, and predictive maintenance.

- Optimization is not necessarily executed on the basis of predictions.
- It reviews the literature on joint maintenance and inventory optimization models for non-repairable parts.

- It classifies literature on the basis of certain criteria.

- It identifies several research gaps.
- It includes only joint maintenance and inventory optimization models.

- It deals with various maintenance strategies.

- It does not distinguish between static and dynamic models. 
Table 1. Cont.

\begin{tabular}{|c|c|c|c|}
\hline Reference & Title & Contributions & Limitations \\
\hline [15] & $\begin{array}{l}\text { Maintenance policy } \\
\text { optimization-literature review } \\
\text { and directions }\end{array}$ & $\begin{array}{l}\text { - It reviews research on optimal } \\
\text { maintenance policy selection } \\
\text { issues associated with } \\
\text { methods used as well as } \\
\text { the applications. } \\
\text { Works are systematically } \\
\text { classified in terms of certainty, } \\
\text { uncertainty, and risk, as well } \\
\text { as in terms of approaches for } \\
\text { optimal maintenance policy. } \\
\text { It identifies a large gap } \\
\text { between academic research } \\
\text { and industrial application. }\end{array}$ & $\begin{array}{l}\text { It deals with various } \\
\text { maintenance strategies. } \\
\text { It does not distinguish } \\
\text { between static and } \\
\text { dynamic models. } \\
\text { It includes limited } \\
\text { real-time models. } \\
\text { Policy optimization is not } \\
\text { necessarily executed on the } \\
\text { basis of predictions. }\end{array}$ \\
\hline [6] & $\begin{array}{l}\text { Industrial maintenance } \\
\text { decision-making: } \\
\text { a systematic literaturereview }\end{array}$ & $\begin{array}{l}\text { - It identifies in literature the } \\
\text { application areas of industrial } \\
\text { maintenance decision-making, } \\
\text { the relationships between } \\
\text { these areas, and the ways in } \\
\text { which authors integrate tools } \\
\text { and methods. } \\
\text { It proposes a framework based } \\
\text { on information from the } \\
\text { literature, which summarizes } \\
\text { the origin and flow of } \\
\text { information used in the } \\
\text { development of models. } \\
\text { It identifies trends towards } \\
\text { joint production and } \\
\text { maintenance optimization and } \\
\text { the utilization autonomous } \\
\text { equipment predictions. }\end{array}$ & $\begin{array}{l}\text { - It deals with various } \\
\text { maintenance strategies. } \\
\text { Decision-making is not } \\
\text { necessarily executed on the } \\
\text { basis of predictions. } \\
\text { It does not distinguish } \\
\text { between static and } \\
\text { dynamic models. }\end{array}$ \\
\hline
\end{tabular}

Table 2. Review protocol.

\begin{tabular}{cl}
\hline Item & \multicolumn{1}{c}{ Description } \\
\hline Keywords & $\begin{array}{l}\text { (data-driven OR real-time OR Internet of Things OR sensor) AND decision } \\
\text { making AND maintenance AND (Industry 4.0 OR smart factory OR } \varnothing)\end{array}$ \\
\hline Inclusion criteria & Papers with decision-making algorithms for maintenance in Industry 4.0 \\
\hline Exclusion criteria & Papers with conceptual approaches \\
\hline Scientific databases & ACM; ArXiv; Emerald; IEEE; ScienceDirect; SpringerLink \\
\hline Time period & January 2013 to March 2021 \\
\hline
\end{tabular}

\section{Analysis and Synthesis}

In this section, we present the analysis and synthesis of the reviewed papers. The process that we followed included the following steps:

1. We structured the literature on data-driven decision-making algorithms for maintenance applications in three areas of contribution, as shown in Table 3. We also assigned each reviewed paper to the respective area.

2. We categorized the methods used in the reviewed papers and we matched them with the areas of contribution as well as with the applications that are presented, as shown in Figures 2 and 3, respectively. It should be noted that some research works incorporate combinations of decision methods, and therefore their references may 
belong to more than one category of methods. Similarly, in some cases, the proposed methods are evaluated in more than one application. Since most of the papers also include a prediction algorithm for deriving prognostic information to be fed into the decision-making algorithm, in this research, we isolated and analyzed only the decision-making algorithms.

3. For each area of contribution, we further specified the categories of methods and the applications of evaluation, while we also performed a thorough discussion and synthesis of the respective papers (Sections 3.1-3.3).

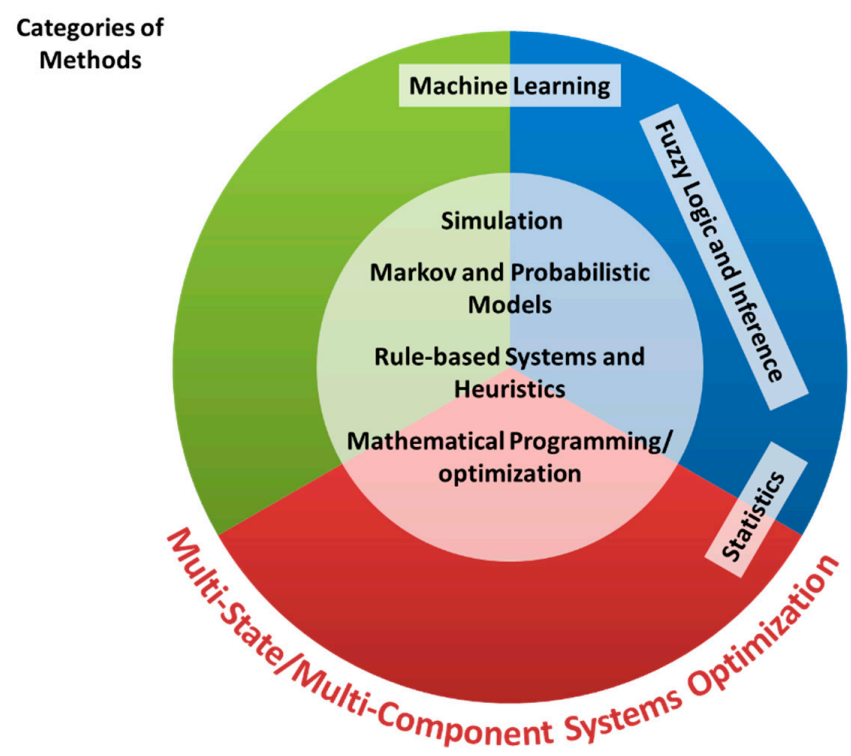

Figure 2. The categories of methods for each area of contribution.

Table 3. Areas of decision-making algorithms in manufacturing maintenance.

\begin{tabular}{ccc}
\hline Area of Contribution & Number of References & References \\
\hline Cost estimation and maintenance planning & 21 & {$[5,20-39]$} \\
Joint scheduling and planning & 14 & 12 \\
Multi-state and multi-component systems optimization & 12 & {$[55-66]$} \\
\hline
\end{tabular}

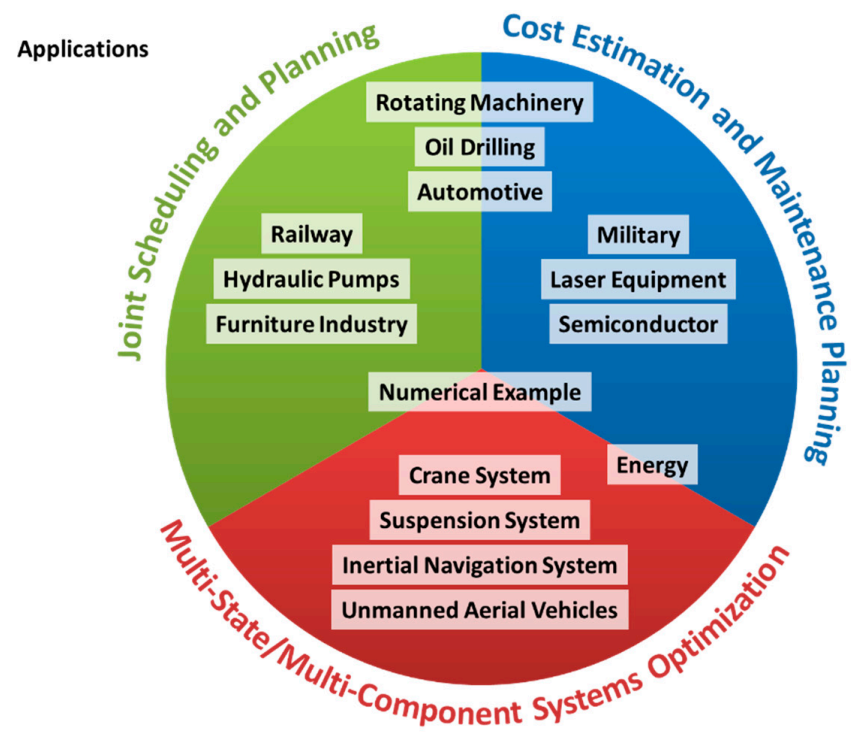

Figure 3. The applications for each area of contribution. 
The overview of the results of this section with respect to the research gaps in the literature of data-driven decision-making algorithms is presented in Table 4. For each area of contribution, we present the main gaps that have not been sufficiently fulfilled by the literature.

Table 4. The research gaps per area of contribution.

\section{Area of Contribution}

Cost estimation and maintenance planning
Research Gaps

(1) Take into account the current level of degradation instead of predictions.

(2) Process batches of data instead of real-time data.

(3) Not utilize data-driven methods leading to problem-specific algorithms.

(4) Rely on the assumption of perfect maintenance or replacement, without considering imperfect maintenance actions with various degrees.

(5) Not take into consideration other factors that affect overall business performance.

(1) Process batches of data instead of real-time data.

(2) Not utilize data-driven methods leading to problem-specific algorithms.

Joint scheduling and planning

(3) Rely on the assumption of perfect maintenance or replacement, without considering imperfect maintenance actions with various degrees.

(4) Include only (usually two) pre-defined objectives.

(5) Assume single component manufacturing systems.

(1) Process batches of data instead of real-time data.

(2) Not utilize data-driven methods leading to problem-specific algorithms.

Multi-state and multi-component systems optimization
(3) Challenges in their implementation in a data-driven manufacturing environment due to their complexity.

(4) Not investigate structural dependence among components.

\subsection{Cost Estimation and Maintenance Planning}

The area of "cost estimation and maintenance planning" includes algorithms that are able to recommend the most appropriate maintenance actions according to the company's policies and the estimations regarding the potential impacts and risks of the candidate actions. In this way, the algorithms aim at minimizing long-term costs, thus enabling the scheduling and planning of mitigating maintenance actions.

Table 5 presents the methods that are used in the papers belonging to this area of contribution, while Table 6 presents the applications in the context of which the proposed algorithms and methods are evaluated. As shown in Table 5, mathematical programming/optimization methods are widely used, while there is also a considerable amount of research on rule-based systems and heuristics as well as on Markov models. As shown in Table 6, applications on rotating machinery and on energy have gathered research interest the most.

The stochastic nature of the degradation process as well as the uncertainty existing in prognostic algorithms leads to high uncertainty also in the decision-making process. For this reason, many papers have tackled these challenges. Hong et al. [20] investigate the influence of stochastic degradation on optimal maintenance decisions. Tang et al. [21] proposed a method for an optimal maintenance policy on the basis of residual life estimation for a slowly degrading system subject to soft failure and condition monitoring. The optimization problem is formulated and solved in a semi-Markov decision process framework in order to minimize the long-run expected average cost. A similar method is proposed for tackling the presence of competing risks (soft and hard failure) in a degrading system [22]. 
Xu et al. [23] proposed a method for optimized replacement decisions on the basis of remaining useful life (RUL) estimation. Wan et al. [24,25] propose a collaborative maintenance planning system that manages information and knowledge to support decision-making in maintenance process planning. Chen et al. [26] propose a fuzzy logic system that allows operators to optimize real-time operation and maintenance scheduling. Yildirim et al. [26] present a maintenance framework that integrates the sensor-driven predictive maintenance technologies with optimal maintenance scheduling models. Fouladirad et al. [27] propose an approach for global optimization of costs for a gradually deteriorating system subject to change in the deterioration rate. Si et al. [28] propose a method that considers not only the expectation of the maintenance cost but also its variability. Lepenioti et al. [29] exploited the recent advancements of machine learning for performing prescriptive analytics on the basis of enterprise and operational data. To do this, the authors applied multi-objective reinforcement learning for providing decisions based upon the predictions of a deep learning algorithm. Hoong et al. [30] propose an algorithm that self-learns an optimal maintenance policy and provides actionable recommendation for each equipment with the use of deep reinforcement learning.

Table 5. Methods for cost estimation and maintenance planning.

\begin{tabular}{cc}
\hline Category of Methods & References \\
\hline Mathematical programming/optimization & {$[5,20,23,26,27,33,35-37]$} \\
Rule-based system and heuristics & {$[5,20,24,31,37]$} \\
Markov and probabilistic models & {$[21-33]$} \\
Simulation & {$[23,31]$} \\
Fuzzy logic and inference & {$[25]$} \\
Machine learning & {$[29,30,32,34]$} \\
\hline
\end{tabular}

Table 6. Evaluation of cost estimation and maintenance planning.

\begin{tabular}{cc}
\hline Applications & References \\
\hline Laser equipment & {$[21,22]$} \\
Rotating machinery & {$[23,26,29-31]$} \\
Semiconductor manufacturing & {$[32]$} \\
Oil drilling & {$[33]$} \\
Energy & {$[20,25,34,37]$} \\
Automotive & {$[33]$} \\
\hline Numerical example & {$[5,27,35,36]$} \\
\hline
\end{tabular}

Several papers take into account the current level of degradation that is derived from the analysis of the indicators measured by sensors instead of predictions about future failure modes, while they usually process batches of data at pre-defined sampling times. However, such approaches are not optimal when decisions are taken under time constraints increasing the maintenance costs of the manufacturing company.

The majority of research works regarding cost estimation and maintenance planning do not adopt a data-driven approach for decision-making, and thus they are limited to specific problems, domains, and industries. That is why mathematical programming/optimization and rule-based systems are the most common categories of methods. Consequently, they cannot be transferred to a different production process with similar challenges in a straightforward way. The most generic approaches aiming at tackling the challenges of the smart factory are presented in [31-34]. Terkaj et al. [31] propose the use of an ontology-based virtual factory in order to enable in situ simulation for assessing the future impact of maintenance planning decisions. Susto et al. [32] proposed an approach with dynamical decision rules in the context of a multiple classifier machine learning methodology aiming at minimizing the expected cost. Bousdekis et al. [33] utilized proactive event-driven computing in maintenance decision-making and represent the 
decision-making process instead of the physical process. Roccheta and Bellani [34] developed a reinforcement learning framework for the prognostic-based optimal management of the maintenance of power grids. The authors of [35] investigated the use of the particle swarm optimization algorithm to quantify the effect of RUL uncertainty on predictive maintenance planning by integrating it with a random sampling-based strategy to select a sequence that performs better for different values of RUL associated with different jobs.

The majority of the algorithms rely on the assumption of perfect maintenance or replacement, without considering imperfect maintenance actions with various degrees. Imperfect maintenance actions for deteriorating systems were considered only in three papers. Do et al. [5] investigated the impacts of imperfect maintenance actions to propose an adaptive maintenance policy that can help to select optimal (perfect or imperfect) maintenance actions at each inspection time according to the remaining useful life (RUL) estimation. Wu et al. [36] propose an optimization model in order to minimize the total cost of imperfect degradation-based maintenance by determining an optimal interval of condition monitoring and the degradation level after imperfect repairs. Bumblauskas et al. [37] propose proactive event-driven decision methods based on Markov Decision Process (MDP) and optimization models for recommending both perfect and imperfect maintenance actions at optimal times with respect to costs.

\subsection{Joint Scheduling and Planning}

As presented in Section 3.1, most cost estimation and maintenance planning approaches do not address factors such as production costs, equipment availability, spare parts inventory, and transportation costs, to name a few. This limitation in prior research paved the way for newer joint scheduling and planning approaches aiming at optimizing jointly manufacturing processes, including maintenance. For example, maintenance planning should be coordinated with spare parts ordering and possible delays, as well as inventory cost management. Table 7 presents the methods that are used in the papers belonging in this area of contribution, while Table 8 presents the applications in the context of which the proposed algorithms and methods are evaluated. As shown in Table 7, mathematical programming/optimization methods are widely used (due to the fact that most of the algorithms are limited to specific problems, domains, and industries), while there is also a considerable amount of research on Markov models. As shown in Table 8, most of the papers in this area evaluate their results in numerical examples, without dealing with case studies. The evaluation of joint scheduling and planning methods in various application domains and real-life case studies is still at its dawn.

Table 7. Methods for joint scheduling and planning.

\begin{tabular}{cc}
\hline Category of Methods & References \\
\hline Mathematical programming/optimization & {$[38,41-44,46,49,50,52-54]$} \\
Rule-based system and heuristics & {$[47-50]$} \\
Markov and probabilistic models & {$[39,45,51,53]$} \\
Simulation & {$[50]$} \\
Machine learning & {$[46]$} \\
\hline
\end{tabular}

Table 8. Evaluation of joint scheduling and planning.

\begin{tabular}{cc}
\hline Applications & References \\
\hline Furniture industry & {$[46]$} \\
Rotating machinery & {$[47,48,52,54]$} \\
Oil drilling & {$[39]$} \\
Hydraulic pumps & {$[49]$} \\
Automotive & {$[43]$} \\
Railway & {$[41]$} \\
Numerical example & {$[38,42,45,50,51,53]$} \\
\hline
\end{tabular}


Similar to the previous area, most of the joint scheduling and planning algorithms rely on the assumption of perfect maintenance or replacement. From the reviewed papers, only [38,39] deal with decision methods capable of recommending both perfect and imperfect maintenance actions. Moreover, apart from [40], the rest of the reviewed papers were involved with single-component systems.

Joint scheduling and planning deal with approaches that optimize predictive maintenance decisions jointly with

- $\quad$ Spare parts inventory ([41-44])

Jiang et al. [41] investigated the impact of inventory deterioration on predictive maintenance decision-making. Van Horenbeek and Pintelon [42] quantified the added value of predictive information (RUL) in dynamic joint maintenance and inventory decisionmaking. Bousdekis et al. [43] propose a proactive event-driven decision model for joint predictive maintenance and spare parts inventory optimization in the frame of the emaintenance concept. Bousdekis et al. [44] propose a proactive event-driven model for joint maintenance and logistics optimization for recommending optimal (perfect or imperfect) maintenance actions and associated spare parts orders along with optimal timing.

- $\quad$ Production planning ([38,45-52])

Kouedeu et al. [38] examined the joint analysis of the optimal production and maintenance planning policies for a manufacturing system subject to random failures and repairs. Jafari and Makis [45] considered the joint optimization of economic manufacturing quantity and maintenance policy. The problem was formulated and solved in the semi-Markov decision process framework in order to minimize the long-run expected average cost per unit time. Cinus et al. [46] propose a decision support system that processes sensor data and Key Performance Indicators (KPIs) using an artificial neural network (ANN)-based knowledge system and integrates the maintenance actions within the weekly production schedule. Mourtzis et al. $[47,48]$ propose an augmented reality mobile application, interfaced with a shop-floor scheduling tool, in order to enable the operator to decide on immediately calling AR remote maintenance or scheduling maintenance tasks for later along with production tasks. Liu et al. [49] present an integrated decision model that coordinates predictive maintenance decisions on the basis of prognostics information with single-machine scheduling decisions so that the total expected cost is minimized. Zhai et al. [50] propose a decision model for predictive maintenance and job shop scheduling for machine deterioration under time-varying operational conditions. Nguyen et al. [51] present a dynamic model for predictive maintenance policy formulated on the basis of partially observable markov decision processes. Mi et al. [52] proposed an integrated decision-making approach supported by digital twin-driven cooperative awareness and interconnection framework.

- $\quad$ Product quality $([53,54])$

Lee and Ni [53] present an approach for determining maintenance and product dispatching policies and the relationship between machine degradation and product quality. They used a Markov decision process for long-term decision-making and integer programming for short-term decision-making. Gu et al. [54] present an algorithm tackling the co-effect between manufacturing system component reliability and product quality. The algorithm results in the optimal maintenance strategy, obtained by optimizing the quality cost, maintenance cost, and interruption cost simultaneously.

- $\quad$ Supplier selection ([44])

Bousdekis et al. [44] propose an approach for real-time, event-driven proactive supplier selection with the use of Markowitz portfolio optimization theory, on the basis of the optimal times for replacement and ordering the spare parts. The algorithm is triggered by real-time, sensor-driven predictions about future failures and future spare parts prices. 


\subsection{Multi-State and Multi-Component Systems Optimization}

In many realistic problems, there are complex multi-component systems with uncertainties in the system reliability structure [55], while the decision-making algorithms for single-component systems are not usually suitable for multi-component systems [6]. To this end, "multi-state and multi-component systems optimization" includes algorithms that allow for the identification of intermediate stages of their health state and that take into account inter-component relations. These relations may be of a stochastic nature, implying that the degradation of one component may probably impact the condition of others. Relations may also be of economic nature, e.g., a positive economic relation implies that it can be more economical if two components are jointly maintained than if done separately.

Table 9 presents the methods that are used in the papers belonging in this area of contribution, while Table 10 presents the applications in the context of which the proposed algorithms and methods are evaluated. As shown in Table 9, mathematical programming/optimization methods are widely used, while there is also a considerable amount of research on rule-based systems and heuristics as well as on Markov and probabilistic models. As shown in Table 10, most of the papers in this area evaluate their results in numerical examples, without dealing with case studies.

Table 9. Methods for multi-state and multi-component systems optimization.

\begin{tabular}{cc}
\hline Category of Methods & References \\
\hline Mathematical programming/optimization & {$[42,56-65]$} \\
Rule-based system and heuristics & {$[51,57,58,61,62]$} \\
Markov and probabilistic models & {$[55,56,58,60]$} \\
Statistics & {$[61]$} \\
Simulation & {$[60]$} \\
\hline
\end{tabular}

Table 10. Evaluation of multi-state and multi-component systems optimization.

\begin{tabular}{cc}
\hline Applications & References \\
\hline Energy & {$[60]$} \\
Crane systems & {$[64]$} \\
Inertial navigation system & {$[57]$} \\
Suspension systems & {$[55]$} \\
Unmanned aerial vehicles & {$[65]$} \\
Numerical example & {$[42,56,58,59,61-63]$} \\
\hline
\end{tabular}

The complexity of multi-state and multi-component systems optimization poses challenges in their scalable and efficient implementation in the context of a data-driven manufacturing environment. Due to this complexity, the reviewed papers usually use algorithms taking advantage of more than one method [56-62], as shown in Table 9.

The dependencies in multi-state and multi-component systems deal with economic dependence, stochastic dependence, or a combination of both. Structural dependence among components was not investigated in the reviewed papers. Below, we describe the reviewed research work for each category of dependence.

- Economic dependence

Le and Tan [63] proposed a multi-state strategy that combines both inspection and continuous monitoring to reduce unnecessary thorough inspection and to improve the system's reliability. An optimal maintenance strategy was derived on the basis of an iterative algorithm to minimize the mean long-run cost-rate. They assumed that the maintenance is imperfect and the degradation is a continuous-time Markov process. Zhou et al. [56] proposed a maintenance optimization method for a multi-state series-parallel system considering economic dependence and state-dependent inspection intervals. The objective function is the average revenue per unit time calculated on the basis of the semi-regenerative theory and the universal generating function (UGF). 
Xia et al. [64] proposed a method of multi-level scheduling in order to predict maintenance requirements according to machine degradation and maintenance opportunity. A global-objective model is used to make the machine-level decision for availabilityeffective and cost-effective maintenance intervals. Nguyen et al. [59] presented an approach for multi-level decision-making for multi-component system with a complex structure. They utilized decision rules for optimally identifying a group of several components as well as a cost-based group improvement factor, taking into account the predictive reliability of the components, the economic dependencies, and the location of the components in the system. Keizer et al. [62] proposed an algorithm for clustering predictive maintenance tasks for systems with both economic dependencies and redundancy with the use of a dynamic programming model.

\section{- Stochastic dependence}

Jiang et al. [57] proposed an approach for providing a predictive maintenance policy for a complex structure by considering not only components' RUL, but also the timing of when system reliability falls below a set threshold. Their approach balances three factors: components importance to the system, risk degree, and detection difficulty. Huynh et al. [58] introduced a multi-level decision-making approach that puts forward an n-component deteriorating system with a k-out-of-n structure. On the basis of the degradation and failure model of the considered k-out-of- $\mathrm{n}$ system, the authors proposed two opportunistic predictive maintenance strategies with different types of maintenance decision-making. Lee and Pan [55] presented a predictive maintenance scheme for complex systems by employing discrete time Markov chain models for modelling multiple degradation processes of components and a Bayesian network (BN) model for predicting system reliability.

- Combination of economic and stochastic dependence

Van Horenbeek and Pintelon [42] presented a dynamic maintenance policy for multicomponent systems that minimizes the long-term mean maintenance cost per unit time. The ability of the maintenance policy to react to changing component deterioration and dependencies within a multi-component system was quantified, and the results showed significant cost savings. Azadeh et al. [60] proposed a model to evaluate the effectiveness of maintenance in multi-component systems using two system performance indicators: reliability and cost. To estimate the reliability and costs of the system, the authors developed the proposed Markovian discrete-event simulation model.

Li et al. [61] focused on the stochastic dependence between components due to the common environment modelled by Lévy copulas. Wang et al. [65] proposed an approach for group maintenance of multi-level systems, in which the reliability of a system is assessed using a Bayesian network (BN) of causes and effect as well as multi-objective programming that is used to optimize plans for joint maintenance of units and components.

\section{Research Agenda for Data-Driven Decision-Making for Industry 4.0 Maintenance Applications}

The specification and documentation of platform Industry 4.0 provide a common view upon which many advancements in industrial technology are based [66]. In this section, we investigate how the nine pillars of Industry 4.0 [67], shown in Figure 4, affect and converge with data-driven decision-making for maintenance. The following subsections describe our propositions for future research in the context of the Industry 4.0 pillars.

Table 11 shows the research agenda of data-driven decision-making algorithms in the context of the Industry 4.0 pillars. For each pillar, we summarize the main propositions for future research in the context of Industry 4.0. 


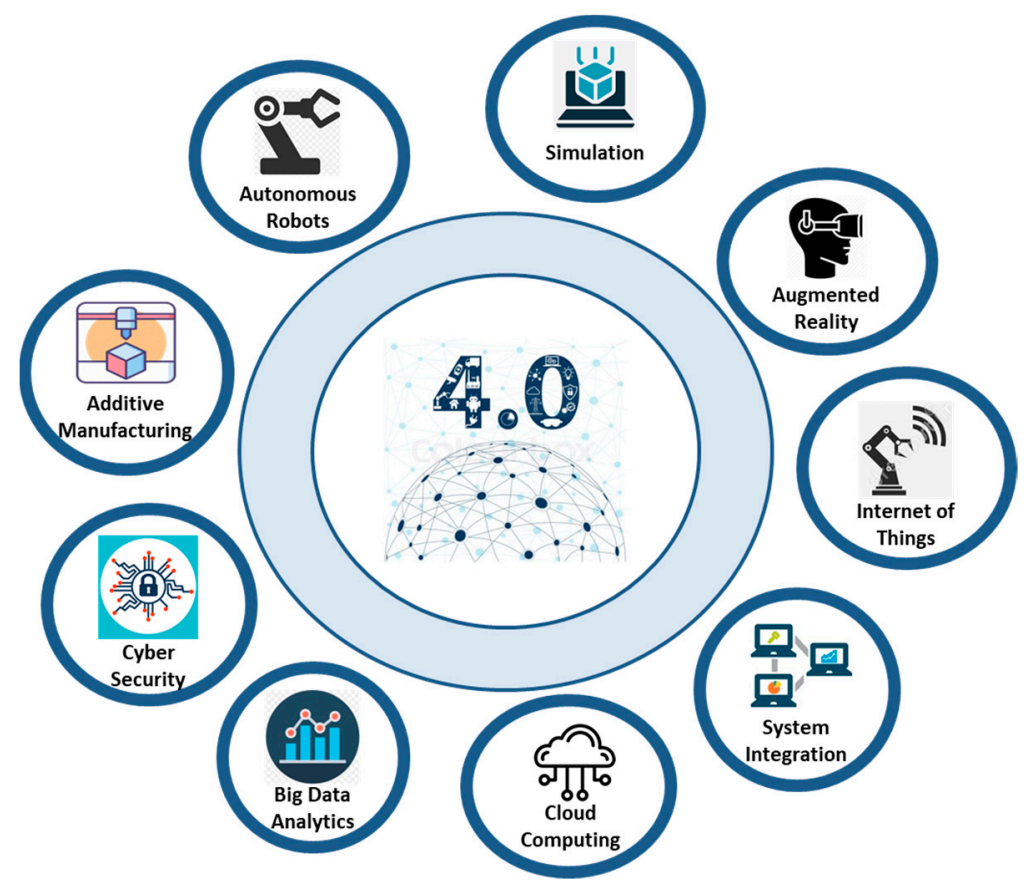

Figure 4. The nine pillars of Industry 4.0.

Table 11. Research agenda of decision-making for Industry 4.0 maintenance applications.

\section{Industry 4.0 Pillars}

Augmented reality

Internet of Things

\section{Future Research Directions}

1. Interface with decision-making algorithms for maintenance applications.

2. Application to the shop-floor during the actual manufacturing operations.

1. Supporting the autonomy of networked manufacturing systems and machines.

2. Eliminating the uncertainty in order to avoid implementing inappropriate autonomous maintenance actions.

3. Fast learning from the shop-floor, exploiting the large availability of data sources.

1. Horizontal and vertical integration according to Industry 4.0 principles.

2. Effective interoperability taking into account RAMI 4.0, CPS architectures, and communication protocols.

3. Decision-making algorithms that take into account the whole context of the manufacturing enterprise.

4. Human as an integral part of system integration.

1. Alignment with the concept of cloud manufacturing.

2. Seamless and modular communication through cloud-based platforms.

3. Communication protocols and standards for guiding the development of future algorithms.

4. Addressing the challenges of reliability, availability, adaptability, and safety.

1. Maintenance decision-making can benefit from prescriptive analytics for big data.

2. Automated data-driven model building in order to represent the decision-making process instead of the physical process.

3. Scalable and efficient algorithms for processing streams of failure predictions and providing meaningful insights.

4. Human feedback mechanisms aiming at improving the decision-making algorithms.

1. Encryption techniques, risk assessment methodologies, and cyber-attack detection and response methods.

2. Modular integration of decision-making software services in a secure and reliable way. 
Table 11. Cont.

\begin{tabular}{|c|c|c|}
\hline Industry 4.0 Pillars & & Future Research Directions \\
\hline Additive manufacturing & 1. & $\begin{array}{l}\text { Decision-making algorithms for manufacturing processes in the context of } \\
\text { additive manufacturing. }\end{array}$ \\
\hline Autonomous robots & $\begin{array}{l}1 . \\
2 . \\
3 . \\
4 .\end{array}$ & $\begin{array}{l}\text { Consider robots as productions resources in decision-making algorithms. } \\
\text { Assign appropriate maintenance tasks to robots. } \\
\text { Decide which tasks will be assigned to robots and which ones to humans. } \\
\text { Uncertainty of decision-making is a significant challenge in automatic action implementation } \\
\text { through autonomous robots. }\end{array}$ \\
\hline Simulation & $\begin{array}{l}1 . \\
2 . \\
3 .\end{array}$ & $\begin{array}{l}\text { Approaches for digital twins in predictive maintenance. } \\
\text { Decision-making algorithms for enhancing the capabilities of digital twins, e.g., by evaluating } \\
\text { different scenarios. } \\
\text { Information fusion methods incorporated in decision-making algorithms for fully exploiting } \\
\text { the available data and knowledge. }\end{array}$ \\
\hline
\end{tabular}

\subsection{Augmented Reality}

Augmented reality (AR) provides a seamless interface combining the real and virtual world aiming at enhancing the collaboration between humans and smart environments [47]. With the advancement of portable devices' processing and visualization capabilities, AR is evolving into an intuitive user interface for displaying information and interacting with machines and services in smart factories $[48,68,69]$.

Although the potential of AR in maintenance operations has been outlined in the literature [70-72], its use as an advanced user interface with decision-making algorithms (e.g., recommending maintenance personnel which mitigating actions to implement and guiding them in how to implement them, aiming at eliminating the impact of a predicted failure) has not been investigated. We found only one paper [47] making use of AR for supporting decisions. Moreover, AR has been used for training purposes; however, its application on the shop-floor during the actual manufacturing and maintenance operations remains a challenge.

\subsection{Internet of Things}

Internet of Things provides a dynamic global network infrastructure with self-configuring capabilities based on standard and interoperable communication protocols where physical and virtual "things" are interconnected and integrated into the information network [73]. In the manufacturing context, the value chain should be intelligent, agile, and networked by integrating physical objects, human factors, intelligent machines, smart sensors, the production process, and production lines together [67]. Since more and more physical objects are connected to the manufacturing network and high-speed transactional data and information is generated [74], scalability is a major challenge in Industry 4.0.

The evolutionary process will lead to networked manufacturing systems with a high degree of autonomy as well as self-optimization capabilities [75]. They will be organized in a decentralized manner, increasing robustness and adaptability [75]. Therefore, the increasing availability of sensors and actuators will result in the need for decisionmaking algorithms capable of supporting the autonomy of networked manufacturing systems. On the other hand, the uncertainty existing in decision-making algorithms for maintenance increases the risk of implementing inappropriate autonomous maintenance actions. To this end, methods and techniques for eliminating the uncertainty and for fast learning from the shop-floor are of utmost importance.

\subsection{System Integration}

System integration increases the value to a system by creating new functionalities through the combination of sub-systems and software applications. The paradigm of Indus- 
try 4.0 is essentially outlined by three dimensions of integration: (a) horizontal integration across the entire value creation network, (b) vertical integration and networked manufacturing systems, and (c) end-to-end engineering across the entire product life cycle [67]. The full digital integration and automation of manufacturing processes in the vertical and horizontal dimension also imply automation of communication and cooperation, especially along standardized processes.

The reviewed papers of the "joint scheduling and planning" area aim at integrating operational with maintenance decision-making in manufacturing processes. However, system integration aspects of decision-making algorithms in terms of interoperability, service communication, modularity, scalability, and flexibility are not discussed. On the other hand, architectures that include decision-making functionalities for maintenance have started to emerge [39,76-80]. Following the trend of diagnostic and prognostic algorithms, decision-making algorithms need to be integrated horizontally and vertically according to the Industry 4.0 principles. In this way, the algorithms will be able to take into account the whole context of the environment (e.g., production plan, supply chain, inventory) by communicating effectively and interchanging data and information with other manufacturing operations. RAMI 4.0 and CPS architectures are significant enablers towards this direction. Finally, integration deals also with the human as an integral part of the manufacturing environment in the sense that there is a cognitive interaction between the human and the system. To this end, the human cyber physical system (H-CPS) concept has arisen [81], which paves the way for the use of emerging technologies implementing human-machine symbiosis.

\subsection{Cloud Computing}

The use of cloud-based architectures and technologies is strongly related to effective systems integration, e.g., with the use of RESTful Application Programming Interfaces (APIs) for accessing services provided by cloud computing vendors. Cloud consumers use APIs as software interfaces to connect and consume resources in various ways, although the optimal or contemporary route is to use a RESTful protocol-based API. To this end, the services implementing data-driven decision-making functionalities should allow seamless and modular communication through cloud-based platforms. This direction needs to be developed in alignment with the concept of cloud manufacturing.

Cloud manufacturing is a smart networked manufacturing model that incorporates cloud computing, aiming at meeting the growing demands for broader global cooperation, knowledge-intensive innovation, and increased market-response agility [82]. Apart from the technological perspective, this will lead to decision-making algorithms facilitating their implementation in a cloud-based computational environment, but also to domain-specific communication protocols and standards for guiding the development of future algorithms requiring high computational power.

On the other hand, this research direction requires addressing the challenges of reliability, availability, adaptability, and safety on machines and processes across spatial boundaries and disparate data sources [83]. In addition, it needs to tackle the privacy and security aspects on the cloud. Therefore, there is the need for robust algorithms that can accurately support decision-making in the presence of uncertainty as well as methods to quantify their confidence in a real-time and computationally demanding environment.

\subsection{Big Data Analytics}

The collection and processing of data from many different sources have significantly enabled the information that is available to engineers and operators in manufacturing facilities. Data management and distribution in the big data environment is critical for achieving self-aware and self-learning machines and for supporting manufacturing decisions. Data analytics is categorized into three main stages characterized by different levels of difficulty, value, and intelligence [84]: (i) descriptive analytics, answering the questions "What has happened?", "Why did it happen?", but also "What is happening now?"; (ii) pre- 
dictive analytics, answering the questions "What will happen?" and "Why will it happen?" in the future; and (iii) prescriptive analytics, answering the questions "What should I do?" and "Why should I do it?".

Although big data analytics has been extensively used for real-time diagnosis and prognosis in the context of predictive maintenance, their utilization in decision-making algorithms is still at its early stages. Since the research interest has been gathered to descriptive and predictive analytics [85], the immaturity of prescriptive analytics has inevitably affected the predictive maintenance decision-making algorithms as well. As we presented in our literature review, the vast majority of the existing predictive maintenance decisionmaking algorithms rely on traditional mathematical programming methods. On the other hand, prescriptive analytics has been realized mainly with domain-specific expert systems or optimization models [86].

However, there is the need for data-driven generic decision-making algorithms representing the decision-making process instead of the physical process. Building physical models of industrial assets and processes is a complex and laborious task that does not necessary exploit the knowledge that can be discovered from data, such as failure patterns or patterns of causes and effects. Moreover, knowledge-based decision-making methods are rather static, not capable of self-adaptation on the basis of emerging data. However, dynamic shop-floor conditions require real-time decision-making, which requires both advanced data infrastructures, e.g., distributed cloud computing for processing and storing data, as well as new functionalities, e.g., computationally efficient, probabilistic algorithms tailored for streaming data and capable of handling uncertainty. Finally, feedback mechanisms should be employed in order to adapt decision-making on the basis of changing conditions such as new operating constraints. Although feedback mechanisms for diagnostic and prognostic algorithms have been well perceived, mechanisms for tracking the recommended actions are still few in number, e.g., [87].

\subsection{Cyber Security}

With the increased connectivity and use of standard communications protocols that come with Industry 4.0, the need to protect critical industrial systems and manufacturing lines from cyber security threats increases significantly. As a result, secure, reliable communications as well as sophisticated identity and access management of machines and users on the basis of a cloud infrastructure are essential [67]. Existing technologies may not be sufficient for industrial applications that have their own safety and security rules and requirements [74]. With respect to decision-making algorithms for maintenance, these cybersecurity techniques and algorithms will enable the efficient and modular integration of decision-making software services with existing diagnostic and prognostic information systems in a secure and reliable way.

\subsection{Additive Manufacturing}

Companies have just begun to adopt additive manufacturing, such as 3-D printing, which they use mostly to prototype and produce individual components. With Industry 4.0 , additive manufacturing methods will be widely used to produce faster and in a more cost-effective way small batches of customized products that offer construction advantages, such as complex, lightweight designs [88]. To this end, a maintenance solution should be to deal with the specific requirements of such products and their respective manufacturing processes. Decision-making algorithms in this context will deal with laser repairing processes, taking into account the contextual background of additive manufacturing.

\subsection{Autonomous Robots}

Autonomous robots are beneficial for manufacturing processes since they can facilitate production tasks that are difficult for humans to perform (e.g., lift heavy tools), need high accuracy and flexibility, constitute boring routines, or even involve the need to access 
dangerous areas $[67,89]$. However, the high automation of production processes poses new challenges in the execution of production tasks.

Autonomous robots constitute additional resources that need to be managed along with the manufacturing equipment and the personnel. Therefore, maintenance decisionmaking algorithms need to take into account the fact that certain maintenance actions may be implemented by autonomous robots, something that affects the scheduling of the maintenance tasks to be performed. These algorithms will assign the appropriate maintenance tasks to humans or robots according to the nature of the task, the knowledge of humans and robots, the trade-off between efficiency of humans and the efficiency of robots for this particular task, the trade-off between the cost of executing the task by a robot or a human, etc. Another important factor is the uncertainty of the prognostic information and thus the suitability of the recommended predictive maintenance actions. Recommendations about maintenance actions with high uncertainty of suitability may be difficult to be addressed automatically by robots that do not have the intelligence to understand the whole context and potential consequences of applying an inappropriate action. On the other hand, the human can ignore the recommendations or react during their execution if they realize that the actions are not suitable.

\subsection{Simulation}

Simulation is a well-established approach in manufacturing environments for avoiding high costs and issues of reliability and safety by the actual implementation of new approaches on the shop-floor. For example, decision-making algorithms for maintenance have utilized such methods for evaluating different scenarios of potential failures and mitigating or corrective actions. However, simulation obtains a completely new meaning in the context of Industry 4.0, which is characterized by the strong connection of the physical and the digital world in which natural and human made systems (physical space) are tightly integrated with computation, communication, and control systems (cyberspace). This concept is named cyber physical systems (CPS).

In the context of Industry 4.0, simulation reaches the next frontier: digital twin. A digital twin is a virtual representation of a physical object generated with digital data. A digital twin can be used to run simulations throughout the design, build, and operation lifecycle [90]. However, its aim is not just digitally representing the physical counterpart. Digital twins that address maintenance operations will need decision-making algorithms capable of generating various scenarios along with their expected impact. To do this, they will need to incorporate information fusion methods in order to automatically or semi-automatically transform information from different sources and different points in time into a representation that provides effective support for human or automated decision-making. Such sources can be sensors, legacy systems, physical models, and expert knowledge. In this way, they will be able to exploit all the available information sources for constructing scenarios and for resulting in the most appropriate ones according to the business objectives and constraints. Up until now, generic approaches for incorporating decision-making algorithms in digital twins addressing predictive maintenance aspects are an unexplored area.

\section{Conclusions}

The availability of data generated by manufacturing automation systems; sophisticated, IoT-enabled machines; and a plethora of sensors challenges existing methods for decision-making in the context of Industry 4.0 maintenance applications. In this paper, we performed a literature review on data-driven decision-making methods for manufacturing applications with a focus on maintenance operations. Our analysis of the literature highlights the emergence of an increasing number of data-driven decision-making methods developed specifically to exploit the plethora of sensor-generated data in the context of Industry 4.0. Coupled with the emergence of cyber-physical systems as well as cloud technologies for processing and storing data, next-generation decision-making for main- 
tenance will be increasingly more responsive and capable of facilitating accurate and proactive decisions.

Author Contributions: Conceptualization K.L.; methodology, K.L., A.B.; analysis and synthesis K.L., A.B., D.A, G.M.; supervision G.M.; project administration G.M. and D.A.; funding acquisition, A.B., D.A., G.M. All authors have read and agreed to the published version of the manuscript.

Funding: Research reported in this article was funded by the European Union's Horizon 2020 Research and Innovation program, grant agreements UPTIME “Unified Predictive Maintenance System" No. 768634.

Data Availability Statement: No significant datasets were analyzed in this study or created to support it.

Conflicts of Interest: The authors declare no conflict of interest. The funders had no role in the design of the study; in the collection, analyses, or interpretation of data; in the writing of the manuscript, or in the decision to publish the results.

\section{References}

1. Huh, J.H.; Lee, H.G. Simulation and Test Bed of a Low-Power Digital Excitation System for Industry 4.0. Processes 2018, 6, 145. [CrossRef]

2. González, I.; Calderón, A.J.; Figueiredo, J.; Sousa, J. A literature survey on open platform communications (OPC) applied to advanced industrial environments. Electronics 2019, 8, 510. [CrossRef]

3. Lucas-Estañ, M.C.; Sepulcre, M.; Raptis, T.P.; Passarella, A.; Conti, M. Emerging trends in hybrid wireless communication and data management for the industry 4.0. Electronics 2018, 7, 400. [CrossRef]

4. Jimenez-Cortadi, A.; Irigoien, I.; Boto, F.; Sierra, B.; Rodriguez, G. Predictive maintenance on the machining process and machine tool. Appl. Sci. 2020, 10, 224. [CrossRef]

5. Do, P.; Voisin, A.; Levrat, E.; Iung, B. A proactive condition-based maintenance strategy with both perfect and imperfect maintenance actions. Reliab. Eng. Syst. Saf. 2015, 133, 22-32. [CrossRef]

6. Ruschel, E.; Santos, E.A.P.; Loures, E.D.F.R. Industrial maintenance decision-making: A systematic literature review. J. Manuf. Syst. 2017, 45, 180-194. [CrossRef]

7. Amruthnath, N.; Gupta, T. A research study on unsupervised machine learning algorithms for early fault detection in predictive maintenance. In Proceedings of the 2018 5th International Conference on Industrial Engineering and Applications (ICIEA), Singapore, 26-28 April 2018; pp. 355-361. [CrossRef]

8. Bousdekis, A.; Lepenioti, K.; Ntalaperas, D.; Vergeti, D.; Apostolou, D.; Boursinos, V. A RAMI 4.0 View of Predictive Maintenance: Software Architecture, Platform and Case Study in Steel Industry. In International Conference on Advanced Information Systems Engineering; Springer: Cham, Switzerland, 2019; pp. 95-106. [CrossRef]

9. Han, Y.; Song, Y.H. Condition monitoring techniques for electrical equipment-a literature survey. IEEE Trans. Power Deliv. 2003, 18, 4-13. [CrossRef]

10. Márquez, F.P.G.; Tobias, A.M.; Pérez, J.M.P.; Papaelias, M. Condition monitoring of wind turbines: Techniques and methods. Renew. Energy 2012, 46, 169-178. [CrossRef]

11. Veldman, J.; Wortmann, H.; Klingenberg, W. Typology of condition based maintenance. J. Qual. Maint. Eng. 2011, 17, 183-202. [CrossRef]

12. Sharma, A.; Yadava, G.S.; Deshmukh, S.G. A literature review and future perspectives on maintenance optimization. J. Qual. Maint. Eng. 2011, 17, 5-25. [CrossRef]

13. Vasili, M.; Hong, T.S.; Ismail, N.; Vasili, M. Maintenance optimization models: A review and analysis. Optimization 2011, 1, 2.

14. Van Horenbeek, A.; Buré, J.; Cattrysse, D.; Pintelon, L.; Vansteenwegen, P. Joint maintenance and inventory optimization systems: A review. Int. J. Prod. Econ. 2013, 143, 499-508. [CrossRef]

15. Ding, S.H.; Kamaruddin, S. Maintenance policy optimization-literature review and directions. Int. J. Adv. Manuf. Technol. 2015, 76, 1263-1283. [CrossRef]

16. Tranfield, D.; Denyer, D.; Smart, P. Towards a methodology for developing evidence-informed management knowledge by means of systematic review. Br. J. Manag. 2003, 14, 207-222. [CrossRef]

17. Galli, A.; Gravina, M.; Moscato, V.; Sperli, G. Deep Learning for HDD health assessment: An application based on LSTM. IEEE Trans. Comput. 2020. [CrossRef]

18. Petrillo, A.; Picariello, A.; Santini, S.; Scarciello, B.; Sperli, G. Model-based vehicular prognostics framework using Big Data architecture. Comput. Ind. 2020, 115, 103177. [CrossRef]

19. Ma, Z.; Ren, Y.; Xiang, X.; Turk, Z. Data-driven decision-making for equipment maintenance. Autom. Constr. 2020, 112. [CrossRef]

20. Hong, H.P.; Zhou, W.; Zhang, S.; Ye, W. Optimal condition-based maintenance decisions for systems with dependent stochastic degradation of components. Reliab. Eng. Syst. Saf. 2014, 121, 276-288. [CrossRef] 
21. Tang, D.; Makis, V.; Jafari, L.; Yu, J. Optimal maintenance policy and residual life estimation for a slowly degrading system subject to condition monitoring. Reliab. Eng. Syst. Saf. 2015, 134, 198-207. [CrossRef]

22. Tang, D.; Yu, J.; Chen, X.; Makis, V. An optimal condition-based maintenance policy for a degrading system subject to the competing risks of soft and hard failure. Comput. Ind. Eng. 2015, 83, 100-110. [CrossRef]

23. Xu, Y.; Zhang, Y.; Zhang, S. Uncertain generalized remaining useful life prediction-driven predictive maintenance decision In Proceedings of the Prognostics and System Health Management Conference (PHM), Beijing, China, 21-23 October 2015; pp. 1-6. [CrossRef]

24. Wan, S.; Gao, J.; Li, D.; Tong, Y.; He, F. Web-based process planning for machine tool maintenance and services. Procedia CIRP 2015, 38, 165-170. [CrossRef]

25. Chen, P.C.; Kezunovic, M. Fuzzy Logic Approach to Predictive Risk Analysis in Distribution Outage Management. IEEE Trans. Smart Grid 2016, 7, 2827-2836. [CrossRef]

26. Yildirim, M.; Sun, X.A.; Gebraeel, N.Z. Sensor-driven condition-based generator maintenance scheduling-Part I: Maintenance problem. IEEE Trans. Power Syst. 2016, 31, 4253-4262. [CrossRef]

27. Fouladirad, M.; Grall, A. On-Line Change Detection and Condition-Based Maintenance for Systems with Unknown Deterioration Parameters. Ima J. Manag. Math. 2014, 25, 139-158. [CrossRef]

28. Si, X.S.; Zhang, Z.X.; Hu, C.H. A Real-Time Variable Cost-Based Maintenance Model. In Data-Driven Remaining Useful Life Prognosis Techniques; Springer: Berlin/Heidelberg, Germany, 2017; pp. 393-404. [CrossRef]

29. Lepenioti, K.; Pertselakis, M.; Bousdekis, A.; Louca, A.; Lampathaki, F.; Apostolou, D.; Mentzas, G.; Anastasiou, S. Machine Learning for Predictive and Prescriptive Analytics of Operational Data in Smart Manufacturing. In Advanced Information Systems Engineering Workshops; Dupuy-Chessa, S., Proper, H.A., Eds.; Lecture Notes in Business Information Processing; Springer International Publishing: Cham, Switzerland, 2020; Volume 382, pp. 5-16. ISBN 978-3-030-49164-2.

30. Hoong Ong, K.S.; Niyato, D.; Yuen, C. Predictive Maintenance for Edge-Based Sensor Networks: A Deep Reinforcement Learning Approach. In Proceedings of the 2020 IEEE 6th World Forum on Internet of Things (WF-IoT), New Orleans, LA, USA, 2-16 June 2020; pp. 1-6.

31. Terkaj, W.; Tolio, T.; Urgo, M. A virtual factory approach for in situ simulation to support production and maintenance planning. CIRP Ann. 2015, 64, 451-454. [CrossRef]

32. Susto, G.A.; Schirru, A.; Pampuri, S.; McLoone, S.; Beghi, A. Machine learning for predictive maintenance: A multiple classifier approach. IEEE Trans. Ind. Inform. 2015, 11, 812-820. [CrossRef]

33. Bousdekis, A.; Papageorgiou, N.; Magoutas, B.; Apostolou, D.; Mentzas, G. Enabling Condition-Based Maintenance Decisions with Proactive Event-driven Computing. Comput. Ind. 2018, 100, 173-183. [CrossRef]

34. Rocchetta, R.; Bellani, L.; Compare, M.; Zio, E.; Patelli, E. A reinforcement learning framework for optimal operation and maintenance of power grids. Appl. Energy 2019, 241, 291-301. [CrossRef]

35. Benaggoune, K.; Meraghni, S.; Ma, J.; Mouss, L.H.; Zerhouni, N. Post Prognostic Decision for Predictive Maintenance Planning with Remaining Useful Life Uncertainty. In Proceedings of the 2020 Prognostics and Health Management Conference (PHMBesan $\sqrt{ }$ Bon), Besancon, France, 4-7 May 2020; pp. 194-199.

36. Wu, F.; Niknam, S.A.; Kobza, J.E. A cost effective degradation-based maintenance strategy under imperfect repair. Reliab. Eng. Syst. Saf. 2015, 144, 234-243. [CrossRef]

37. Bumblauskas, D.; Gemmill, D.; Igou, A.; Anzengruber, J. Smart Maintenance Decision Support Systems (SMDSS) based on corporate big data analytics. Expert Syst. Appl. 2017, 90, 303-317. [CrossRef]

38. Kouedeu, A.F.; Kenné, J.P.; Dejax, P.; Songmene, V.; Polotski, V. Production and maintenance planning for a failure-prone deteriorating manufacturing system: A hierarchical control approach. Int. J. Adv. Manuf. Technol. 2015, 76, 1607-1619. [CrossRef]

39. Bousdekis, A.; Mentzas, G. A Proactive Model for Joint Maintenance and Logistics Optimization in the Frame of Industrial Internet of Things. In Operational Research in the Digital Era-ICT Challenges; Springer: Cham, Switzerland, 2019; pp. 23-45. [CrossRef]

40. Van Horenbeek, A.; Pintelon, L. A joint predictive maintenance and inventory policy. In Engineering Asset Management-Systems, Professional Practices and Certification; Springer: Cham, Switzerland, 2015; pp. 387-399. [CrossRef]

41. Jiang, Y.; Chen, M.; Zhou, D. Joint optimization of preventive maintenance and inventory policies for multi-unit systems subject to deteriorating spare part inventory. J. Manuf. Syst. 2015, 35, 191-205. [CrossRef]

42. Van Horenbeek, A.; Pintelon, L. A dynamic predictive maintenance policy for complex multi-component systems. Reliab. Eng. Syst. Saf. 2013, 120, 39-50. [CrossRef]

43. Bousdekis, A.; Papageorgiou, N.; Magoutas, B.; Apostolou, D.; Mentzas, G. A proactive event-driven decision model for joint equipment predictive maintenance and spare parts inventory optimization. Procedia CIRP 2017, 59, 184-189. [CrossRef]

44. Bousdekis, A.; Papageorgiou, N.; Magoutas, B.; Apostolou, D.; Mentzas, G. A Framework for Integrated Proactive Maintenance Decision Making and Supplier Selection. In IFIP International Conference on Advances in Production Management Systems; Springer: Cham, Switzerland, 2017; pp. 416-424. [CrossRef]

45. Jafari, L.; Makis, V. Joint optimal lot sizing and preventive maintenance policy for a production facility subject to condition monitoring. Int. J. Prod. Econ. 2015, 169, 156-168. [CrossRef] 
46. Cinus, M.; Confalonieri, M.; Barni, A.; Valente, A. An ANN Based Decision Support System Fostering Production Plan Optimization Through Preventive Maintenance Management. In Advances in Neural Networks; Springer: Cham, Switzerland, 2016; pp. 447-455. [CrossRef]

47. Mourtzis, D.; Vlachou, E.; Zogopoulos, V.; Fotini, X. Integrated production and maintenance scheduling through machine monitoring and augmented reality: An Industry 4.0 approach. In IFIP International Conference on Advances in Production Management Systems; Springer: Cham, Switzerland, 2017; pp. 354-362. [CrossRef]

48. Mourtzis, D.; Vlachou, A.; Zogopoulos, V. Cloud-Based Augmented Reality Remote Maintenance through Shop-Floor Monitoring: A Product-Service System Approach. J. Manuf. Sci. Eng. 2017, 139, 061011. [CrossRef]

49. Liu, Q.; Dong, M.; Chen, F.F. Single-machine-based joint optimization of predictive maintenance planning and production scheduling. Robot. Comput. Integr. Manuf. 2018, 51, 238-247. [CrossRef]

50. Zhai, S.; Riess, A.; Reinhart, G. Formulation and Solution for the Predictive Maintenance Integrated Job Shop Scheduling Problem. In Proceedings of the 2019 IEEE International Conference on Prognostics and Health Management (ICPHM), San Francisco, CA, USA, 17-20 June 2019; pp. 1-8. [CrossRef]

51. Nguyen, K.T.; Do, P.; Huynh, K.T.; Bérenguer, C.; Grall, A. Joint optimization of monitoring quality and replacement decisions in condition-based maintenance. Reliab. Eng. Syst. Saf. 2019, 189, 177-195. [CrossRef]

52. Mi, S.; Feng, Y.; Zheng, H.; Wang, Y.; Gao, Y.; Tan, J. Prediction maintenance integrated decision-making approach supported by digital twin-driven cooperative awareness and interconnection framework. J. Manuf. Syst. 2021, 58, 329-345. [CrossRef]

53. Lee, S.; Ni, J. Joint decision making for maintenance and production scheduling of production systems. Int. J. Adv. Manuf. Technol. 2013, 66, 1135-1146. [CrossRef]

54. Gu, C.; He, Y.; Han, X.; Chen, Z. Product quality oriented predictive maintenance strategy for manufacturing systems. In Proceedings of the Prognostics and System Health Management Conference (PHM), Harbin, China, 9-12 July 2017; pp. 1-7. [CrossRef]

55. Lee, D.; Pan, R. Predictive maintenance of complex system with multi-level reliability structure. Int. J. Prod. Res. 2017, 55, 4785-4801. [CrossRef]

56. Zhou, Y.; Zhang, Z.; Lin, T.R.; Ma, L. Maintenance optimisation of a multi-state series-parallel system considering economic dependence and state-dependent inspection intervals. Reliab. Eng. Syst. Saf. 2013, 111, 248-259. [CrossRef]

57. Jiang, X.; Duan, F.; Tian, H.; Wei, X. Optimization of reliability centered predictive maintenance scheme for inertial navigation system. Reliab. Eng. Syst. Saf. 2015, 140, 208-217. [CrossRef]

58. Huynh, K.T.; Barros, A.; Bérenguer, C. Multi-Level Decision-Making for the Predictive Maintenance of k-Out-of-n Deteriorating Systems. IEEE Trans. Reliab. 2015, 64, 94-117. [CrossRef]

59. Nguyen, K.A.; Do, P.; Grall, A. Multi-level predictive maintenance for multi-component systems. Reliab. Eng. Syst. Saf. 2015, 144, 83-94. [CrossRef]

60. Azadeh, A.; Asadzadeh, S.M.; Salehi, N.; Firoozi, M. Condition-based maintenance effectiveness for series-parallel power generation system-A combined Markovian simulation model. Reliab. Eng. Syst. Saf. 2015, 142, 357-368. [CrossRef]

61. Li, H.; Deloux, E.; Dieulle, L. A condition-based maintenance policy for multi-component systems with Lévy copulas dependence. Reliab. Eng. Syst. Saf. 2016, 149, 44-55. [CrossRef]

62. Keizer, M.C.O.; Teunter, R.H.; Veldman, J. Clustering condition-based maintenance for systems with redundancy and economic dependencies. Eur. J. Oper. Res. 2016, 251, 531-540. [CrossRef]

63. Le, M.D.; Tan, C.M. Optimal maintenance strategy of deteriorating system under imperfect maintenance and inspection using mixed inspection scheduling. Reliab. Eng. Syst. Saf. 2013, 113, 21-29. [CrossRef]

64. Xia, T.; Xi, L.; Zhou, X.; Lee, J. Condition-based maintenance for intelligent monitored series system with independent machine failure modes. Int. J. Prod. Res. 2013, 51, 4585-4596. [CrossRef]

65. Wang, X.; Zhang, Y.; Wang, L.; Wang, J.; Lu, J. Maintenance grouping optimization with system multi-level information based on BN lifetime prediction model. J. Manuf. Syst. 2019, 50, 201-211. [CrossRef]

66. Rossit, D.A.; Tohmé, F.; Frutos, M. Industry 4.0: Smart Scheduling. Int. J. Prod. Res. 2019, 57, 3802-3813. [CrossRef]

67. Vaidya, S.; Ambad, P.; Bhosle, S. Industry 4.0-A Glimpse. Procedia Manuf. 2018, 20, 233-238. [CrossRef]

68. Rabah, S.; Assila, A.; Khouri, E.; Maier, F.; Ababsa, F.; Bourny, V.; Maier, P.; Mérienne, F. Towards Improving the Future of Manufacturing through Digital Twin and Augmented Reality Technologies. Procedia Manuf. 2018, 17, 460-467. [CrossRef]

69. Wang, X.; Yew, A.W.W.; Ong, S.K.; Nee, A.Y.C. Enhancing Smart Shop Floor Management with Ubiquitous Augmented Reality. Int. J. Prod. Res. 2020, 58, 2352-2367. [CrossRef]

70. Oliveira, R.; Farinha, J.T.; Fonseca, I.; Barbosa, F.M. Augmented Reality System for Maintenance of High-Voltage Systems. In Proceedings of the 2016 51st International Universities Power Engineering Conference (UPEC), Coimbra, Portugal, 6-9 September 2016; pp. 1-5.

71. Lorenz, M.; Knopp, S.; Klimant, P. Industrial Augmented Reality: Requirements for an Augmented Reality Maintenance Worker Support System. In Proceedings of the 2018 IEEE International Symposium on Mixed and Augmented Reality Adjunct (ISMAR-Adjunct), Munich, Germany, 16-20 October 2018; pp. 151-153.

72. Loizeau, Q.; Danglade, F.; Ababsa, F.; Merienne, F. Evaluating Added Value of Augmented Reality to Assist Aeronautical Maintenance Workers-Experimentation on On-field Use Case. In Virtual Reality and Augmented Reality; Bourdot, P., Interrante, V., Nedel, L., Magnenat-Thalmann, N., Zachmann, G., Eds.; Lecture Notes in Computer Science; Springer International Publishing: Cham, Switzerland, 2019; Volume 11883, pp. 151-169. ISBN 978-3-030-31907-6. 
73. Xu, L.D.; He, W.; Li, S. Internet of Things in Industries: A Survey. IEEE Trans. Ind. Inf. 2014, 10, 2233-2243. [CrossRef]

74. Xu, L.D.; Xu, E.L.; Li, L. Industry 4.0: State of the Art and Future Trends. Int. J. Prod. Res. 2018, 56, 2941-2962. [CrossRef]

75. Jeschke, S.; Brecher, C.; Meisen, T.; Özdemir, D.; Eschert, T. Industrial Internet of Things and Cyber Manufacturing Systems. In Industrial Internet of Things; Jeschke, S., Brecher, C., Song, H., Rawat, D.B., Eds.; Springer Series in Wireless Technology; Springer International Publishing: Cham, Switzerland, 2017; pp. 3-19. ISBN 978-3-319-42558-0.

76. Schmidt, B.; Wang, L. Cloud-Enhanced Predictive Maintenance. Int. J. Adv. Manuf. Technol. 2018, 99, 5-13. [CrossRef]

77. Makris, S.; Nikolakis, N.; Dimoulas, K.; Papavasileiou, A.; Ippolito, M. SERENA: Versatile Plug-and-Play Platform Enabling Remote Predictive Maintenance. In Enterprise Interoperability; Zelm, M., Jaekel, F.-W., Doumeingts, G., Wollschlaeger, M., Eds.; John Wiley \& Sons, Inc.: Hoboken, NJ, USA, 2018; pp. 277-283. ISBN 978-1-119-56403-4.

78. Reina, A.; Cho, S.-J.; May, G.; Coscia, E.; Cassina, J.; Kiritsis, D. Maintenance Planning Support Tool Based on Condition Monitoring with Semantic Modeling of Systems. In Enterprise Interoperability; Zelm, M., Jaekel, F.-W., Doumeingts, G., Wollschlaeger, M., Eds.; John Wiley \& Sons, Inc.: Hoboken, NJ, USA, 2018; pp. 271-276. ISBN 978-1-119-56403-4.

79. Cerquitelli, T.; Bowden, D.; Marguglio, A.; Morabito, L.; Napione, C.; Panicucci, S.; Nikolakis, N.; Makris, S.; Coppo, G.; Andolina, S.; et al. A Fog Computing Approach for Predictive Maintenance. In Advanced Information Systems Engineering Workshops; Proper, H.A., Stirna, J., Eds.; Lecture Notes in Business Information Processing; Springer International Publishing: Cham, Switzerland, 2019; Volume 349, pp. 139-147. ISBN 978-3-030-20947-6.

80. Ansari, F.; Glawar, R.; Nemeth, T. PriMa: A Prescriptive Maintenance Model for Cyber-Physical Production Systems. Int. J. Comput. Integr. Manuf. 2019, 32, 482-503. [CrossRef]

81. Romero, D.; Bernus, P.; Noran, O.; Stahre, J.; Fast-Berglund, Å. The Operator 4.0: Human Cyber-Physical Systems \& Adaptive Automation towards Human-Automation Symbiosis Work Systems. In Advances in Production Management Systems. Initiatives for a Sustainable World; Nääs, I., Vendrametto, O., Mendes Reis, J., Gonçalves, R.F., Silva, M.T., von Cieminski, G., Kiritsis, D., Eds.; IFIP Advances in Information and Communication Technology; Springer International Publishing: Cham, Switzerland, 2016; Volume 488, pp. 677-686. ISBN 978-3-319-51132-0.

82. Ren, L.; Zhang, L.; Wang, L.; Tao, F.; Chai, X. Cloud Manufacturing: Key Characteristics and Applications. Int. J. Comput. Integr. Manuf. 2017, 30, 501-515. [CrossRef]

83. Wang, J.; Zhang, L.; Duan, L.; Gao, R.X. A New Paradigm of Cloud-Based Predictive Maintenance for Intelligent Manufacturing. J. Intell. Manuf. 2017, 28, 1125-1137. [CrossRef]

84. Frazzetto, D.; Nielsen, T.D.; Pedersen, T.B.; Siksnys, L. Prescriptive Analytics: A Survey of Emerging Trends and Technologies. $V L D B$ J. 2019, 28, 575-595. [CrossRef]

85. Hagerty, J. Planning Guide for Data and Analytics; Gartner Inc.: Stamford, CO, USA, 2017; Volume 13.

86. Lepenioti, K.; Bousdekis, A.; Apostolou, D.; Mentzas, G. Prescriptive Analytics: Literature Review and Research Challenges. Int. J. Inf. Manag. 2020, 50, 57-70. [CrossRef]

87. Warnell, G.; Waytowich, N.; Lawhern, V.; Stone, P. Deep Tamer: Interactive Agent Shaping in High-Dimensional State Spaces. In Proceedings of the AAAI Conference on Artificial Intelligence, New Orleans, LA, USA, 2-7 February 2018 ; Volume 32.

88. Sandengen, O.C.; Estensen, L.A.; Rødseth, H.; Schjølberg, P. High Performance Manufacturing-An Innovative Contribution towards Industry 4.0. In Proceedings of the 6th International Workshop of Advanced Manufacturing and Automation, Manchester, UK, 10-11 November 2016.

89. Chukwuekwe, D.O.; Schjoelberg, P.; Roedseth, H.; Stuber, A. Reliable, Robust and Resilient Systems: Towards Development of a Predictive Maintenance Concept within the Industry 4.0 Environment. In Proceedings of the EFNMS Euro maintenance conference, Athens, Greece, 30 May-1 June 2016.

90. Tao, F.; Zhang, M. Digital Twin Shop-Floor: A New Shop-Floor Paradigm towards Smart Manufacturing. IEEE Access 2017, 5, 20418-20427. [CrossRef] 